\title{
recillunds
}

Revista Cientifica Mundo de la Investigación y el Conocimiento

\author{
Margarita Alexandra Ontano Moreno a ; Ana Isabel Mejía Velasteguí ${ }^{\text {b; }}$ \\ Marietta Elizabeth Avilés Arroyo ${ }^{c}$
}

Uso del ácido tranexámico en hemorragia obstétrica

Use of tranexamic acid in obstetric hemorrhage

Revista Científica Mundo de la Investigación y el Conocimiento. Vol. 3 núm. 4., diciembre, ISSN: 2588-073X, 2019, pp. 194-211

DOI: $10.26820 /$ recimundo/3.(4).diciembre.2019.194-211

URL: http://recimundo.com/index.php/es/article/view/659

Código UNESCO: 3205 Medicina Interna

Tipo de Investigación: Artículo de Revisión

(C) RECIMUNDO; Editorial Saberes del Conocimiento, 2019

Recibido: 15/09/2019

Aceptado: 23/11/2019

Publicado: 30/12/2019

Correspondencia: karinaborjaa@gmail.com

a. Magister en Docencia y Gerencia en Educación Superior; Magister en Ginecología Infante Juvenil y Salud Reproductiva del Adolescente; Obstetriz, Universidad de Guayaquil, Guayaquil, Ecuador; karinaborjaa@gmail.com

b. Especialista en Ginecología y Obstetricia; Médico; Universidad de Guayaquil, Guayaquil, Ecuador; karitonaty@ hotmail.com

c. Especialista en Ginecología y Obstetricia; Doctora en Medicina y Cirugía; Universidad de Guayaquil; Guayaquil, Ecuador; lujam898989@gmail.com 


\section{Uso del ácido tranexámico en hemorragia obstétrica}

Vol. 3, núm. 4., (2019)

Margarita Alexandra Ontano Moreno; Ana Isabel Mejía Velasteguí;

Marietta Elizabeth Avilés Arroyo

\section{RESUMEN}

Las hemorragias durante el embarazo, postparto inmediato y puerperio tardío son complicaciones que traen una morbididad y mortalidad a la paciente y el feto. No existe una definición universal de la hemorragia obstétrica, porque no se ha establecido claramente, lo que constituye una pérdida sanguínea excesiva. La pérdida de volumen sanguíneo a una velocidad superior a 150 $\mathrm{ml} / \mathrm{min}$ (que en 20 minutos causaría la pérdida del 50\% del volumen), que puede presentarse durante el estado grávido o puerperal, que supera los $500 \mathrm{ml}$, postparto o $1000 \mathrm{ml}$ post cesárea. El ácido tranexámico es un derivado de la lisina utilizado como hemostático gracias a sus propiedades antifibrinolíticas. Inhibe la activación del plasminógeno al bloquear los ligando de la lisina de esta molécula. Ejerce su efecto antifibrinolítico mediante el bloqueo reversible y competitivo de los receptores de lisina en las moléculas de PLG, impidiendo la unión de éste a la fibrina. Reduce la activación del PLG a PL con lo cual se inhibe la degradación de la fibrina, favoreciendo así la hemostasia secundaria. El ácido tranexámico no presenta pruebas de teratogenicidad o de otros efectos secundarios tanto en la madre como el feto, evidenciando epidemiológicamente que no habría contraindicación para su uso durante el embarazo en caso de existir hemorragias. Respecto a la seguridad de su uso tanto para la madre como para el recién nacido, los ensayos clínicos no se reportan eventos adversos serios y significativos para el uso de ácido tranexámico; destacando evidencia que señalan eventos adversos leves con significancia estadística en molestias transitorias tales como cefalea náuseas, vómitos, diarrea y fotopsias. La metodología usada es descriptiva, con un enfoque documental, es decir, revisar fuentes disponibles en la red, como google académico, con contenido oportuno y relevante desde el punto de vista científico y actualizado que enriquezca el análisis del tema planteado en este artículo.

Palabras Claves: Hemorragia Obstetricia; Ácido Tranexámico; Plasminógeno; Antifibrinolíticas; Pérdida Sanguínea Excesiva; PLG. 


\section{Uso del ácido tranexámico en hemorragia obstétrica}

Vol. 3, núm. 4., (2019)

Margarita Alexandra Ontano Moreno; Ana Isabel Mejía Velasteguí;

Marietta Elizabeth Avilés Arroyo

\section{ABSTRACT}

Hemorrhages during pregnancy, immediate postpartum and late puerperium are complications that bring morbidity and mortality to the patient and the fetus. There is no universal definition of obstetric hemorrhage, because it has not been clearly established, which constitutes excessive blood loss. Loss of blood volume at a rate greater than $150 \mathrm{ml} / \mathrm{min}$ (which in 20 minutes would cause the loss of $50 \%$ of the volume), which can occur during the gravid or puerperal state, which exceeds $500 \mathrm{ml}$, postpartum or $1000 \mathrm{ml}$ post Caesarean section. Tranexamic acid is a derivative of lysine used as hemostatic thanks to its antifibrinolytic properties. It inhibits plasminogen activation by blocking the lysine ligands of this molecule. It exerts its antifibrinolytic effect by reversing and competitive blocking of lysine receptors in PLG molecules, preventing its binding to fibrin. Reduces the activation of PLG to PL which inhibits fibrin degradation, thus favoring secondary hemostasis. Tranexamic acid does not show evidence of teratogenicity or other side effects in both the mother and the fetus, epidemiologically proving that there would be no contraindication for use during pregnancy in case of bleeding. Regarding the safety of its use for both the mother and the newborn, clinical trials do not report serious and significant adverse events for the use of tranexamic acid; highlighting evidence indicating mild adverse events with statistical significance in transient discomfort such as headache nausea, vomiting, diarrhea and photopsies. The methodology used is descriptive, with a documentary approach that is, reviewing sources available on the web, such as google scholar, with timely and relevant content from the scientific and updated point of view that enriches the analysis of the topic raised in this article.

Keywords: Obstetrics Hemorrhage; Tranexamic Acid; Plasminogen; Antifibrinolytics; Excessive Blood Loss; PLG. 


\section{Uso del ácido tranexámico en hemorragia obstétrica}

Vol. 3, núm. 4., (2019)

Margarita Alexandra Ontano Moreno; Ana Isabel Mejía Velasteguí;

Marietta Elizabeth Avilés Arroyo

\section{Introducción.}

Las hemorragias durante el embarazo, postparto inmediato y puerperio tardío son complicaciones que traen una morbididad y mortalidad a la paciente y el feto. Informes internacionales reportan una incidencia de $1.2 \%$ de hemorragias durante el embarazo, $8.3 \%$ en el post-parto inmediato con parto vaginal y un $5.7 \%$ pos-cesárea y $0.2 \%$ en el puerperio tardío (Murray, 2019). Sin embargo, la incidencia de transfusión fue de 1,14\%. Los procedimientos quirúrgicos obstétricos se asocian con sangrado post operatorio, el cual es habitualmente autolimitado. No obstante, en pacientes con desórdenes hereditarios de la hemostasia, procedimientos invasivos menores pueden precipitar sangrados prolongados. Estos sangrados excesivos son alarmantes tanto para el paciente como para el tratante, y pueden alargar el procedimiento, comprometer la cicatrización y predisponer a infecciones.

Cuando los tejidos se dañan, los vasos sanguíneos pueden romperse e inmediatamente desencadenar el mecanismo hemostático, consistente en vasoconstricción, formación tapón plaquetario e inicio coagulación; dando lugar a una red estable de fibrina. La fibrinólisis es el mecanismo fisiológico que disuelve coágulos, mantiene a los vasos sanguíneos permeables y da inicio a la remodelación del tejido dañado; planteándose así que su bloqueo favorecería la hemostasia. El uso de inhibidores de la fibrinólisis empezó hace varias décadas y hoy en el mercado, existen sólo productos sintéticos, principalmente el ácido epsilón-aminocaproico y el ácido tranexámico.

El ácido tranexámico es un medicamento antifibrinolítico ampliamente usado en medicina. En el parto, su uso inhibiría la actividad fibrinolítica aumentada, ejerciendo un efecto 


\section{Uso del ácido tranexámico en hemorragia obstétrica}

Vol. 3, núm. 4., (2019)

Margarita Alexandra Ontano Moreno; Ana Isabel Mejía Velasteguí;

Marietta Elizabeth Avilés Arroyo

clínico de prevención primaria en contra del sangrado, por lo que en aquellas situaciones de potencial hemorragia materna, previo consentimiento informado materno, recomendamos su uso. Ante un sangrado durante el parto, debiera usarse pues los potenciales peligros no evidenciados serían muy menores a los beneficios observados en su uso clínico como se evidenciará a continuación.

\section{Metodología.}

Esta investigación está dirigida al estudio del "Uso del ácido tranexámico en hemorragia obstétrica”. Para realizarlo se usó una metodología tipo descriptiva, con un enfoque documental, es decir, revisar fuentes disponibles en la red, como google académico, con contenido oportuno y relevante desde el punto de vista científico para dar respuesta a lo tratado en el presente artículo y que sirvan de inspiración para realizar otros proyectos. Las mismas pueden ser consultadas al final, en la bibliografía.

\section{Resultados.}

\section{Hemorragia obstetricia}

No existe una definición universal de la hemorragia obstétrica, porque no se ha establecido claramente, lo que constituye una pérdida sanguínea excesiva. La pérdida de volumen sanguíneo a una velocidad superior a $150 \mathrm{ml} / \mathrm{min}$ (que en 20 minutos causaría la pérdida del $50 \%$ del volumen), que puede presentarse durante el estado grávido o puerperal, que supera los $500 \mathrm{ml}$, postparto o $1000 \mathrm{ml}$ post cesárea (Toledo-Asturizaga, 2014). Criterios de diagnósticos: 


\section{Uso del ácido tranexámico en hemorragia obstétrica}

Vol. 3, núm. 4., (2019)

Margarita Alexandra Ontano Moreno; Ana Isabel Mejía Velasteguí;

Marietta Elizabeth Avilés Arroyo

- Perdida mayor al 25\% de la volemia. (50\% de la volemia en 3 horas).

- Descenso del hematocrito 10 o más puntos.

- Pérdida aproximada de $150 \mathrm{ml} /$ minuto en 20 minutos.

- Descenso de la hemoglobina $4 \mathrm{gr} / \mathrm{dl}$.

- Cambios hemodinámicos que desencadena presencia de síntomas: debilidad, vértigo, síncope y /o signos: hipotensión, taquicardia u oliguria. Estos datos están presentes cuando la paciente ha paciente ha perdido una cantidad importante de sangre.

\section{Clasificación}

Antes de parto:

- Placenta previa: la hemorragia genital se caracteriza por ser indolora, con expulsión de sangre líquida de cantidad variable, roja rutilante, que aparece en forma brusca e inesperada, la mayoría de las veces en reposo e incluso durante el sueño. Se presenta al final del segundo trimestre, habitualmente sin actividad uterina.

- Desprendimiento de placenta: síndrome hemorrágico que se presenta por desprendimiento total o parcial de la placenta normalmente insertada.

- Ruptura uterina: se dividen en dehiscencia (rotura de cicatriz previa) y rotura de útero sin cicatriz previa (por maniobras obstétricas, traumatismos, trabajo de parto prolongado con desproporción feto-materna). El principal factor de riesgo es el antecedente de una cesárea previa $0.2 \%$, luego de dos cesáreas previas el riesgo se eleva a 3.7\%. Los signos clínicos son: dolor abdominal, dolor a la palpación, pérdida de bienestar fetal, palpación 


\section{Uso del ácido tranexámico en hemorragia obstétrica}

Vol. 3, núm. 4., (2019)

Margarita Alexandra Ontano Moreno; Ana Isabel Mejía Velasteguí;

Marietta Elizabeth Avilés Arroyo

fácil de partes fetales, muerte fetal, hemorragia vaginal, colapso circulatorio materno; taquicardia, hipotensión.

- Vasa previa: es una condición rara cuya falta de diagnóstico puede ocasionar una mortalidad fetal del $60 \%$. Ocurre cuando existe una inserción velamentosa del cordón, que atraviesa las membranas en el segmento inferior del útero por delante de la presentación fetal. Clínicamente se evidencia sangrado genital que ocurre después de la ruptura de membranas, con un útero relajado, en presencia de riesgo de pérdida del bienestar fetal que no guarda relación con la cuantía de la hemorragia (ToledoAsturizaga, 2014). Ante la sospecha se debe analizar la sangre vaginal para determinar si existe hemoglobina fetal y se debe proceder a interrupción inmediata del embarazo por la vía más expedita.

En el puerperio:

- Precoz 50\% (Primeras 24 horas) Atonía uterina (50 a 60\%)

- Traumatismo cérvico-vaginal (20 a 30\%)

- Retención de restos ovulares (10\%)

- Trastornos adherenciales placentarios

- Inversión uterina

Tardío (Entre las 24 horas y la 6ta. semana)

- Retención de restos ovulares.

- Endomiometritis. 


\section{Uso del ácido tranexámico en hemorragia obstétrica}

Vol. 3, núm. 4., (2019)

Margarita Alexandra Ontano Moreno; Ana Isabel Mejía Velasteguí; Marietta Elizabeth Avilés Arroyo

- Involución anormal del lecho placentario.

- Dehiscencia de la histerorrafia.

Secundarias: coagulopatías congénitas, adquiridas

- Coagulación intravascular diseminada

- Coagulopatía pos transfusional

- Sepsis intrauterina

- Pre eclampsia /HELLP

- Óbito fetal

Tratamiento medicamentoso (de primera línea) (Vergara-Soto, 2018)

- Oxitócicos: oxitocina $10 \mathrm{U}$, endovenoso lento, constituye una de las recomendaciones preventivas, mantener con $20 \mathrm{U}$ en $500 \mathrm{ml}$ de solución glucosada.

- Maleato de ergometrina $0,2 \mathrm{mg}$ IM y reevaluar a los 10 minutos.

- Carbetocina 100 ug, IV previa liberación de receptores con 200 ml de solución fisiológica a infusión continua por 5 a 6 minutos.

- Misoprostol 800 a 1000 Ug, vía rectal. (Recomendación-C).

- El ácido tranexámico se ha sugerido en caso de atonía refractaria o sangrado persistente secundario a trauma genital. 


\section{Uso del ácido tranexámico en hemorragia obstétrica}

Vol. 3, núm. 4., (2019)

Margarita Alexandra Ontano Moreno; Ana Isabel Mejía Velasteguí;

Marietta Elizabeth Avilés Arroyo

Tratamiento intervencionista (de segunda línea)

- Masaje uterino bi manual, una mano a través de la vagina en puño presionando la pared anterior del útero y la otra supra umbilical sobre cara posterior del cuerpo uterino.

- Taponamiento uterino (efectivo en el $84 \%$ de los casos), el dispositivo más utilizado fue el catéter Sengstaken Blakemore, Barki (Karlsson, 2009).

- La colocación de un balón (Cobra Cordis 5F) a la arteria iliaca interna a través de la femoral común, siguiendo la técnica de Seldinger, a una presión1-2atm., se insufla y se deja por 24 horas a 48 horas, con profilaxis antibiótica, este procedimiento es seguro, efectivo y se puede realizar en pacientes inestables. Es importante contar con el apoyo de un radiólogo experimentado (Torre, Junio 2018).

- Indumentaria antichoque, no existe estudios randomizados que lo recomienden, la posibilidad de beneficiar al paciente está en disminuir la pérdida de sangre 200 $\mathrm{ml}$ aproximadamente lo que no diferencia determina diferencia de la conducta quirúrgica a seguir.

\section{Tratamiento radiológico}

En paciente estable, la embolización de la arteria uterina alcanza una efectividad del 90\%, preservando la fertilidad y recuperando las menstruaciones en un $100 \%$ después de un parto. Puede presentarse complicaciones como: dolor, fiebre, embolismo pulmonar, infección pélvica hasta necrosis de útero y vejiga (0 a 10\%) (Vergara-Soto, 2018). 


\section{Uso del ácido tranexámico en hemorragia obstétrica}

Vol. 3, núm. 4., (2019)

Margarita Alexandra Ontano Moreno; Ana Isabel Mejía Velasteguí;

Marietta Elizabeth Avilés Arroyo

\section{Ácido tranexámico}

El ácido tranexámico es un derivado de la lisina utilizado como hemostático gracias a sus propiedades antifibrinolíticas. Inhibe la activación del plasminógeno al bloquear los ligandos de la lisina de esta molécula (Morgado-Carrasco, 2017). Ejerce su efecto antifibrinolítico mediante el bloqueo reversible y competitivo de los receptores de lisina en las moléculas de PLG, impidiendo la unión de éste a la fibrina. Reduce la activación del PLG a PL con lo cual se inhibe la degradación de la fibrina, favoreciendo así la hemostasia secundaria. La reducción de la actividad de PL obtenida por el uso del ácido tranexámico, es útil en condiciones de elevada actividad fibrinolítica local y sistémica, así como en la mayoría de los trastornos de la coagulación, en que existe una formación de coágulo con una red de fibrina anormal debida a disminución y retraso en la generación de trombina.

Puede administrarse por vía oral, endovenosa o tópica. La dosis endovenosa generalmente es de 0,5 a 1 gramo $(10 \mathrm{mg} / \mathrm{kg}$ de peso corporal $)$, en tres a cuatro tomas al día. Por vía oral, la dosis es de 1 a 1,5 gramos ( 15 a $20 \mathrm{mg} / \mathrm{kg}$ de peso corporal, tres a cuatro veces al día, no excediendo los 4 gramos/día. En caso de cirugía, la primera dosis endovenosa se administra inmediatamente antes de empezar. Sin embargo, si la primera dosis se administra por vía oral, debería suministrarse dos horas antes de la intervención. Puede utilizarse la misma dosis para niños y adultos.

En general el ácido tranexámico es bien tolerado, siendo los eventos adversos habitualmente leves y consistentes con molestias gastrointestinales destacando la presencia de náuseas y diarrea. La inyección endovenosa rápida puede provocar mareos e hipotensión; por lo 


\section{Uso del ácido tranexámico en hemorragia obstétrica}

Vol. 3, núm. 4., (2019)

Margarita Alexandra Ontano Moreno; Ana Isabel Mejía Velasteguí;

Marietta Elizabeth Avilés Arroyo

tanto, se recomienda no administrar el ácido tranexámico a un ritmo mayor de $100 \mathrm{mg} / \mathrm{minuto}$.

Experiencias en relación al uso del ácido tranexámico en dosis mayores a las habituales y en condiciones clínicas particulares, han reportado eventos adversos graves e infrecuentes tales como trombosis, insuficiencia renal y convulsiones. En la actualidad, se consideran contraindicaciones absolutas para el uso:

1. Trombosis venosa o arterial aguda.

2. Hematuria macroscópica originada en el tracto urinario superior.

3. Hemorragia subaracnoidea excepto tratamiento "ultraprecoz" con AT previo a la intervención neuroquirúrgica del aneurisma.

4. Uso de concentrados complejo protrombina en dosis alta y repetida y/o uso de FEIBA (contiene los factores II, IX y $\mathrm{X}$ principalmente no activados, así como el Factor VII activado; el antígeno del Factor VIII coagulante).

\section{Uso del ácido tranexámico en obstetricia}

\section{Embarazo}

Es infrecuente la ocurrencia de problemas hemorrágicos en mujeres embarazadas con trastornos de coagulación congénitos incluso severos como la enfermedad de von Willebrand tipo 3, no obstante su frecuencia es mayor que la población de gestantes sin defectos de coagulación. El ácido tranexámico no presenta pruebas de teratogenicidad o de otros efectos 


\section{Uso del ácido tranexámico en hemorragia obstétrica}

Vol. 3, núm. 4., (2019)

Margarita Alexandra Ontano Moreno; Ana Isabel Mejía Velasteguí; Marietta Elizabeth Avilés Arroyo

secundarios tanto en la madre como el feto evidenciando epidemiológicamente que no habría contraindicación para su uso durante el embarazo en caso de existir hemorragias.

Parto

La hemorragia es una complicación grave del parto, considerándose como la principal causa de muerte materna en el mundo, y una causa importante de morbilidad dado que el $12 \%$ de los sobrevivientes tendrán anemia severa subsecuente (Tisné, 2013) . La pérdida de sangre que se produce durante el parto por cesárea es aproximadamente dos veces más que la cantidad perdida durante el parto vaginal; y aproximadamente el $6 \%$ de estas pacientes requieren transfusiones de sangre. Las mujeres que requieren transfusión de sangre se enfrentan a los riesgos que ésta conlleva, incluyendo la transmisión de infecciones.

Durante el proceso del parto, tanto vaginal como en la cesárea, producto de la injuria vascular se puede producir hemorragia, con la consecuente pérdida de factores de la coagulación, la que puede ser agravada durante el alumbramiento, debido activación del sistema fibrinolítico a niveles pre gestacionales, por la separación placentaria. Lo anterior plantea el uso de ácido tranexámico, no sólo en situaciones de sangrado durante el parto sino también para prevención primaria de la hemorragia antes del nacimiento.

En la línea de la prevención primaria de la hemorragia durante el parto, hay evidencia de ensayos clínicos, que avalan la eficacia y seguridad de una única dosis de ácido tranexámico, para reducir el sangrado tanto en el parto vaginal, como en la cesárea. En cesárea sin factores de riesgo para sangrado, dicha pérdida hemática es menor en un rango de 14,1\% a 52,6\% al usarlo versus placebo, además se reporta una reducción en la frecuencia hemorragia obstétrica (pérdida 


\section{Uso del ácido tranexámico en hemorragia obstétrica}

Vol. 3, núm. 4., (2019)

Margarita Alexandra Ontano Moreno; Ana Isabel Mejía Velasteguí;

Marietta Elizabeth Avilés Arroyo

hemática mayor a $500 \mathrm{ml}$ y/o $400 \mathrm{ml}$ ) en porcentajes variables de 11,1\% a 18\% (Muñoz, Julio 2011). Del mismo modo reportan $25 \%$ menos de transfusiones con el uso de ácido tranexámico respecto a placebo.

Respecto a la seguridad de su uso tanto para la madre como para el recién nacido, los ensayos clínicos no se reportan eventos adversos serios y significativos para el uso de ácido tranexámico; destacando evidencia que señalan eventos adversos leves con significancia estadística en molestias transitorias tales como cefalea náuseas, vómitos, diarrea y fotopsias (Caballero, Febrero 2017). Las gestantes que padecen trastornos hemostáticos congénitos deberían recibir una atención adecuada en colaboración con centros de tratamiento especializado en el manejo de coagulopatías.

El tratamiento durante el parto vaginal o la cesárea debería adaptarse a la paciente en cuestión y podría incluir el uso ácido tranexámico. En el caso de varios trastornos de la coagulación graves, por ejemplo enfermedad Von Willebrand tipo 3 (Tisné, 2013), es necesario administrar concentrado de factor; pero si también se administra este ácido puede reducirse la cantidad de concentrado y la duración de su administración sirviendo de terapia complementaria con ácido tranexámico en estas pacientes.

En la línea del manejo del sangrado después del parto vaginal, usando ácido tranexámico en una dosis inicial de 4 gramos seguida de la infusión endovenosa de 1 gramo/hora durante seis horas, en casos de pérdida hemática mayor a 800 ml se logró una reducción en las medianas de pérdida hemática (ácido tranexámico: $170 \mathrm{ml}$, control: $221 \mathrm{ml}$ ), tiempo duración sangrado (a 30 minutos de iniciada la hemorragia se detuvo sangrado ácido tranexámico: 63\%, control: 46\%), 


\section{Uso del ácido tranexámico en hemorragia obstétrica}

Vol. 3, núm. 4., (2019)

Margarita Alexandra Ontano Moreno; Ana Isabel Mejía Velasteguí; Marietta Elizabeth Avilés Arroyo

cantidad de glóbulos rojos transfundidos (total unidades transfundidas ácido tranexámico: 28, control: 62) e incidencia de disminución concentración de hemoglobina en más de 4 gr/dl (AT: 25\%, control: 43\%). La detención del sangrado posterior al uso de útero retractores y transfusión de concentrados de glóbulos rojos se reveló para ácido tranexámico 93\% en cambio para control fue de $79 \%(\mathrm{p}=0,03)$ (Tisné, 2013).

Un ensayo clínico controlado evaluó la seguridad y la efectividad de la administración temprana de ácido tranexámico en la mortalidad, el requerimiento de histerectomía y otros resultados relevantes, en las mujeres con hemorragia post-parto. Se aplicó a mujeres mayores de 16 años con diagnóstico clínico de hemorragia post-parto posterior a parto vaginal o cesárea. Se consideró hemorragia post-parto al sangrado estimado mayor a 500 mililitros (ml) en caso de parto vaginal, de $1000 \mathrm{ml}$ posterior a cesárea, o de cualquier magnitud suficiente como para producir inestabilidad hemodinámica (Grillo, Junio 2017).

El procedimiento utilizado fue el siguiente: ácido tranexámico 1 gramo (g) a concentración de 100 miligramos $(\mathrm{mg})$ por $\mathrm{ml}$, administrado a una velocidad aproximada de $1 \mathrm{ml} /$ minuto. Si el sangrado continuó posterior a 30 minutos o se detuvo y reinició en las primeras 24 horas de la primera dosis, se administró una segunda dosis. El grupo control recibió placebo a la misma frecuencia. Con base en este ensayo clínico se pudo establecer que (Grillo, Junio 2017), el uso de ácido tranexámico no redujo la frecuencia de muerte por cualquier causa o la necesidad de histerectomía en las primeras 42 horas (RR 0.97, IC 95\% 0.87 a 1.09), al igual que tampoco modificó la frecuencia de muerte por cualquier etiología (RR 0.88, IC 95\% 0.74 a 1.05) (Tisné, 2013). 


\section{Uso del ácido tranexámico en hemorragia obstétrica}

Vol. 3, núm. 4., (2019)

Margarita Alexandra Ontano Moreno; Ana Isabel Mejía Velasteguí;

Marietta Elizabeth Avilés Arroyo

Por otra parte, las pacientes asignadas a recibir ácido tranexámico requirieron con menor frecuencia laparotomía para detener el sangrado (Grillo, Junio 2017) (RR 0.64, IC 95\% 0.49 a 0.85) a expensas de una mayor frecuencia en el uso de suturas hemostáticas (RR 1.19 IC 95\% 1.01 a 1.41). La frecuencia de eventos tromboembólicos no fue diferente entre los grupos (RR 0.88 IC $95 \% 0.54$ a 1.43), al igual que tampoco lo fue la frecuencia de complicaciones (falla renal RR 1.09 IC 95\% 0.85 a 1.39; falla cardiaca RR 0.95 IC 95\% 0.73 a 1.23; falla respiratoria RR 0.87 IC 95\% 0.67 a 1.12; falla hepática RR 0.96 IC 95\% 0.58 a 1.60; sepsis RR 0.97 IC $95 \%$ 0.79 a 1.19 o de convulsiones RR 0.76 IC 95\% 0.49 a 1.20) (Karlsson, 2009).

\section{Embarazo en mujeres con trastornos hemorrágicos}

Las mujeres que se sospeche padezcan un trastorno hemorrágico o sean portadoras deberían someterse a pruebas diagnósticas antes de embarazarse a fin de permitir una asesoría previa a la concepción y un control temprano del embarazo adecuados. Esto es de suma importancia para mujeres con trastornos hemorrágicos graves o para aquellas que pudieran engendrar un bebé que pudiera resultar gravemente afectado, como las portadoras de hemofilia. La asesoría previa a la concepción tiene dos ventajas (Andran, 2009):

- Ofrece a las mujeres y sus familiares información adecuada sobre las implicaciones genéticas de su trastorno, las opciones reproductivas disponibles y las opciones de diagnóstico prenatal.

- Permite planear el embarazo y establecer cómo y dónde puede controlarse mejor. Otros aspectos de la atención previa a la concepción incluyen la inmunización contra la 


\section{Uso del ácido tranexámico en hemorragia obstétrica}

Vol. 3, núm. 4., (2019)

Margarita Alexandra Ontano Moreno; Ana Isabel Mejía Velasteguí; Marietta Elizabeth Avilés Arroyo

hepatitis A y B para quienes posiblemente requieran transfusiones de sangre y asesoría general sobre temas como suplementos de ácido fólico.

Las mujeres con trastornos hemorrágicos corren el riesgo de padecer HPP primaria (pérdida de sangre de más de $500 \mathrm{ml}$ durante las primeras 24 horas posteriores al parto) y secundaria (sangrado excesivo entre 24 horas y 6 semanas después del parto), especialmente quienes padecen trastornos graves. Los hematomas perineales/vaginales son una complicación poco común del parto vaginal, pero es más probable que se presenten en mujeres con trastornos hemorrágicos, particularmente después de partos vaginales quirúrgicos. Puede usarse ácido tranexámico para la prevención y el control de HPP secundarias. Los anticonceptivos orales combinados, de no ser contraindicados, también constituyen una opción para evitar el sangrado excesivo durante los últimos días del periodo posparto.

\section{Conclusiones.}

El plan de acción en el manejo de la hemorragia obstétrica debe ser contextualizado en cada uno de los hospitales que atiendan mujeres embarazadas. El uso de componentes sanguíneos y terapia hemostática farmacológica se consideran terapéuticas con indicaciones precisas, ambas con ventajas, desventajas, efectos colaterales.

En la prevención de la hemorragia post parto el conocimiento de la existencia de factores de riesgo es de gran ayuda a la hora de prevenirla, pero hay que recordar que $2 / 3$ de los casos se dan en mujeres sin ninguno de estos factores. Es por ello que debemos actuar de forma sistemática y universal en la fase de alumbramiento de todos los partos mediante una conducta activa, que puede resumirse en los siguientes pasos: 


\section{Uso del ácido tranexámico en hemorragia obstétrica}

Vol. 3, núm. 4., (2019)

Margarita Alexandra Ontano Moreno; Ana Isabel Mejía Velasteguí;

Marietta Elizabeth Avilés Arroyo

- Administración de un uterotónico, siendo de elección la oxitocina.

- Clampaje y sección precoz del cordón umbilical.

- Tracción suave y mantenida del mismo.

- Masaje uterino tras alumbramiento.

El uso de inhibidores de la fibrinólisis empezó hace varias décadas y hoy en el mercado, existen sólo productos sintéticos, principalmente el ácido epsilón-aminocaproico y el ácido tranexámico. El ácido tranexámico es un medicamento que favorece la hemostasia secundaria, impidiendo la hemorragia. En la actualidad es importante en cirugía y traumatismo para reducir la pérdida de sangre y la necesidad de transfusión. En general el ácido tranexámico es bien tolerado, siendo los eventos adversos habitualmente leves y consistentes con molestias gastrointestinales destacando la presencia de náuseas y diarrea. La inyección endovenosa rápida puede provocar mareos e hipotensión; por lo tanto, se recomienda no administrar el ácido tranexámico a un ritmo mayor de 100 mg/minuto. Experiencias en relación al uso del ácido tranexámico en dosis mayores a las habituales y en condiciones clínicas particulares, han reportado eventos adversos graves e infrecuentes tales como trombosis, insuficiencia renal y convulsiones.

\section{Bibliografía.}

Andran, R. . (2009). Salud reproductiva en las mujeres con trastorno de la coagulación. Federación Mundial de la Hemofilia $N^{\circ}$ 48, 1 - 17.

Caballero, J. (Febrero 2017). Ácido tranexámico: evidencia científica y su traducción en la práctica clínica. Revista Chilena de Cir ugía Vol.69 Nº 1 , https://scielo.conicyt.cl.

Grillo, C. (Junio 2017). Obstetricia. Revista Colombiana de Obstetricia y Ginecología, https://www.fecolsog.org/obstetricia. 


\section{Uso del ácido tranexámico en hemorragia obstétrica}

Vol. 3, núm. 4., (2019)

Margarita Alexandra Ontano Moreno; Ana Isabel Mejía Velasteguí;

Marietta Elizabeth Avilés Arroyo

Karlsson, H. (2009). Hemorragia postparto. Anales SIS San Navarra Vol.32 $N^{\circ} .1$, http://scielo.isciii.es.

Morgado-Carrasco. (2017). Tratamiento oral del melasma: nueva evidencia a favor del uso del a' cido tranexámico. Piel Barcelona Volumen $12 N^{\circ} 3,1-5$.

Muñoz, A. (Julio 2011). El ácido tranexámico disminuye la mortalidad del shock hemorrágico traumático. Medicina Intensiva Vol.35 N5, http://scielo.isciii.es.

Murray, N. (2019). Manejo de una paciente embarazada con déficit congénito de Factor VII leve. Revista chilena de obstetricia y ginecología Vol.84 N², https://scielo.conicyt.cl.

Tisné, L. (2013). Ácido tranexámico en obstetricia y ginecología. Revista de Obstetricia y Ginecología Hospital Santiago Oriente Volumen $8 N^{\circ} 3,143$ - 156 .

Toledo-Asturizaga. (2014). HEMORRAGIA OBSTÉTRICA. Revista Médica La Paz Volumen $20 N^{\circ} 2$, http://www.scielo.org.bo.

Torre, T. D. (Junio 2018). Hemoderivados versus fármacos hemostáticos en la hemorragia obstétrica. ANESTESIA EN GINECO-OBSTETRICIA Volumen 41 N 1, 29 - 31.

Vergara-Soto. (2018). Hemorrágia obstetricia: primer caso reportado en Chile. ARS Medica Volumen $43 N^{\circ}$ 2, 11 - 18 .

$$
\text { (c) } \frac{1}{\mathrm{BY} N \mathrm{NC} \text { SA }}
$$

RECONOCIMIENTO-NOCOMERCIAL-COMPARTIRIGUAL

CC BY-NC-SA

ESTA LICENCIA PERMITE A OTROS ENTREMEZCLAR, AJUSTAR Y CONSTRUIR A PARTIR DE SU OBRA CON FINES NO COMERCIALES, SIEMPRE Y CUANDO LE RECONOZCAN LA AUTORÍA Y SUS NUEVAS CREACIONES ESTÉN BAJO UNA LICENCIA CON LOS MISMOS TÉRMINOS. 